\title{
CONCERNING THE SEPARABILITY OF CERTAIN LOCALLY CONNECTED METRIC SPACES
}

\author{
F. BURTON JONES
}

If a connected metric space $S$ is locally separable, then $S$ is separable. ${ }^{1}$ If a connected, locally connected, metric space $S$ is locally peripherally separable, then $S$ is separable. ${ }^{2}$ Furthermore if a connected, locally connected, complete metric space $S$ satisfies certain "flatness" conditions, it is known to be separable. ${ }^{3}$ These "flatness" conditions are rather strong and involve both im kleinen and im grossen properties, which makes application rather awkward in some cases. If, however, this space $S$ contains no skew curve ${ }^{4}$ of type 1 , then $S$ has a certain amount of "flatness," but not quite enough to imply separability as can be seen from the following example. Let $S$ consist of the points of the 2-sphere, distance being redefined as follows: (1) if the points $X$ and $Y$ of $S$ lie on the same great circle through the poles, then $d(X, Y)$ is the ordinary distance on the sphere but (2) if the points lie on different great circles through the poles, then $d(X, Y)$ is the sum of the ordinary distances from each point to the same pole, using the pole which gives the smaller sum. The space $S$ is a connected, locally connected, complete metric space which contains no skew curve of type 1 but $S$ is not separable. Furthermore, $S$ contains no cut point. However, if this last condition is strengthened slightly, separability follows as is seen in the following theorem.

Theorem 1. Let $S$ denote a locally connected, complete metric space such that no pair of points cuts $S$. If $S$ contains no skew curve of type 1 , then $S$ is separable.

Proof. Suppose, on the contrary, that $S$ is not separable. Let $T_{0}$

Presented to the Society, November 24, 1945; received by the editors November 16, 1945.

1 Paul Alexandroff, Über die Metrization der im kleinen kompakten topologischen Räume, Math. Ann. vol. 92 (1924) pp. 294-301. Also W. Sierpinski, Sur les espaces metriques localement separables, Fund. Math. vol. 21 (1933) pp. 107-113.

${ }^{2}$ F. B. Jones, $A$ theorem concerning locally peripherally separable spaces, Bull. Amer. Math. Soc. vol. 41 (1935) pp. 437-439.

${ }^{3} \mathrm{~F}$. B. Jones, Concerning certain topologically flat spaces, Trans. Amer. Math Soc. vol. 42 (1937) pp. 53-93, Theorem 31. Also F. B. Jones, Bull. Amer. Math. Soc. Abstract 47-1-93.

"Kuratowski in his paper, Sur le problème des courbes gauches en Topologie, Fund. Math. vol. 15 (1930) pp. 271-283, defined two "skew curves." One of type 1 is topologically equivalent to the sum of three simple triods each two of which intersect precisely at their end points. 
denote a simple triod in $S$ and let $M_{1}=T_{0}$. Let $T_{1}$ denote a simple triod in $S$ having only its end points in $M_{1}$, and let $M_{2}=T_{0}+T_{1}$. Let $T_{2}$ denote a simple triod in $S$ having only its end points in $M_{2}$, and let $M_{3}=T_{0}+T_{1}+T_{2}$. This process may be continued, so that if $z$ is an ordinal less than $\Omega_{1}$, then (a) $M_{z}=\overline{\Sigma T_{\eta}}, 0 \leqq \eta<z$, (b) $M_{z}$ is a separable (hence proper) subcontinuum of $S$, and (c) $T_{z}$ is a simple triod having only its end points in $M_{z}$. Hence $T_{1}, T_{2}, T_{3}, \ldots, T_{z}, \ldots$ is an uncountable sequence $\alpha$ of simple triods such that no two of them have a point in common which is not an end point of one of them. For each $z<\Omega_{1}$, let $d_{z}$ denote the smallest of the distances: $d(A, \operatorname{arc} B O C)$, $d(B, \operatorname{arc} A O C)$, and $d(C, \operatorname{arc} A O B)$, where $A, B$, and $C$ are the end points and $O$ is the emanation point of $T_{z}$. Let $H_{1}$ denote the set of all simple triods $T$ such that for some $z, T$ is $T_{z}$ of $\alpha$ and each end point of $T$ lies together with a point of $M_{1}$ in a connected domain of diameter less than $d_{z} / 5$.

Suppose that $H_{1}$ is uncountable. There exists a positive number $\epsilon$ such that for uncountably many different ordinals $z<\Omega_{1}, T_{z}$ belongs to $H_{1}$ and $1.1 \epsilon>d_{z} / 5>\epsilon$. But since $M_{1}$ is separable, there exist three distinct points $X_{1}, X_{2}$, and $X_{3}$ and three ordinals $\alpha<\beta<\gamma<\Omega_{1}$ such that (1) $T_{\alpha}, T_{\beta}$, and $T_{\gamma}$ each belong to $H_{1},(2)$ for each $\xi, \xi=\alpha, \beta, \gamma$, $1.1 \epsilon>d_{\xi} / 5>\epsilon$, and (3) for each $\xi, \xi=\alpha, \beta, \gamma$, and each $i, i=1,2,3$, there exists a connected domain $D_{\xi i}$ which contains $X_{i}$ and an end point of $T_{\xi}$, and whose diameter is less than $d_{\xi} / 5$. Now for each $\xi$, $\xi=\alpha, \beta, \gamma$, let $O_{\xi}$ denote the emanation point of $T_{\xi}$. From (2), (3), the definition of $d_{z}$, and the triangle axiom on the distance function, it follows that the connected domains, $D_{1}=\Sigma D_{\xi 1}, D_{2}=\Sigma D_{\xi 2}$, and $D_{3}=\Sigma D_{\xi 3}$, are mutually exclusive and neither $\bar{D}_{1}, \bar{D}_{2}$, nor $\bar{D}_{3}$ contains either $O_{\alpha}, O_{\beta}$, or $O_{\gamma}$. Because of the restricted way in which the triods may intersect, no point outside of $D_{1}+D_{2}+D_{3}$ lies in more than one of the triods $T_{\alpha}, T_{\beta}$, and $T_{\gamma}$. But $T_{\alpha} \cdot D_{1}$ may be joined to $T_{\beta} \cdot D_{1}$ by an arc in $D_{1} ; T_{\alpha} \cdot D_{1}$ may be joined to $T_{\gamma} \cdot D_{1}$ by an arc in $D_{1} ; T_{\alpha} \cdot D_{2}$ may be joined to $T_{\beta} \cdot D_{2}$ by an arc in $D_{2}$; and so on; and in the sum of these arcs together with $T_{\alpha}+T_{\beta}+T_{\gamma}$ there exists a skew curve of type 1 . So the assumption that $H_{1}$ is uncountable leads to a contradiction. Hence $H_{1}$ is countable.

Let $z_{2}$ denote the smallest ordinal such that if $z \geqq z_{2}, T_{z}$ of $\alpha$ does not belong to $H_{1}$. Evidently $z_{2}<\Omega_{1}$. Let $H_{2}$ denote the set of all triods $T$ such that for some $z, T$ is $T_{z}$ of $\alpha$ and each end point of $T$ lies together with a point of $M_{z}$ in a connected domain of diameter less than $d_{z} / 5$. The collection $H_{2}$ is countable. Let $z_{3}$ denote the smallest ordinal such that if $z \geqq z_{3}, T_{z}$ of $\alpha$ does not belong to $H_{2}$. Evidently $z_{3}<\Omega_{1}$. Let $H_{3}$ denote the set of all triods $T$ such that for some $z, T$ is $T$, of $\alpha$ and 
each end point of $T$ lies together with a point of $M_{z_{3}}$ in a connected domain of diameter less than $d_{z} / 5$. The collection $H_{3}$ is countable. Continue this process, so that for each natural number $n, H_{n}$ is defined and countable. There exists an ordinal number $z<\Omega_{1}$ such that for each $n, z>z_{n}$. Let $\bar{z}$ denote the first such ordinal. Clearly, $T_{z}$ of $\alpha$ does not belong to $H_{n}$ for any $n$. Let $D_{1}, D_{2}$, and $D_{3}$ denote three mutually exclusive connected domains covering respectively the end points of $T_{\mathrm{z}}$ such that each has a diameter less than $d_{\mathrm{i}}$. Since $M_{1} \subset M_{2} \subset M_{3} \subset \cdots \subset M_{2} \subset \cdots$ and $T_{3}$ has its end points in $M_{3}$, there exists an integer $i$ such that each of the domains, $D_{1}, D_{2}$, and $D_{3}$, intersects $M_{z_{i}}$. Hence $T_{i}$ belongs to $H_{i}$. This is a contradiction.

TheOREM 2. Let $S$ denote a locally connected, complete metric space such that no pair of points cuts $S$. If $S$ does not contain uncountably many skew curves of type 1 , then $S$ is separable.

Theorem 2 may be established by the argument for Theorem 1, taking for $T_{0}$ the closure of the set consisting of all points $X$ such that $X$ belongs to a skew curve of type 1 lying in $S$. The connectedness of $M_{z}$ was not used in the argument.

Comment. This result (Theorem 1) cannot be extended to complete Moore spaces. ${ }^{5}$ For a locally connected complete Moore space exists which is not cut by any pair of its points and which contains no skew curve of type 1 but which nevertheless is not separable. ${ }^{6}$ Furthermore, a separable such space exists which is not completely (perfectly) separable and hence is not metric. ${ }^{7}$ The relation between Moore and metric spaces (in this connection) is shown in Theorem 3.

THEOREM 3. Let $M$ denote a locally connected, complete Moore space such that (1) no pair of points cuts $M$ and (2) $M$ contains no skew curve of type 1. In order thai $M$ be metric it is necessary and sufficient that $M$ be completely (perfectly) separable.

Proof. Since any metric, complete Moore space is a complete metric space ${ }^{8}$ and any separable metric space is completely separable, the necessity of the condition follows at once from Theorem 1 . Since a

B R. L. Moore, Foundations of point set theory, Amer. Math. Soc. Colloqiuum Publications, vol 13,1932. Hereinafter this book will be referred to as Foundations. A complete Moore space is a space satisfying Axioms 0 and 1 of Foundations.

- R. L. Moore, Concerning separability, Proc. Nat. Acad. Sci. U.S.A. vol. 28 (1942) pp. 56-58, Example 1.

${ }^{7}$ Ibid. Example 2.

$8 \mathrm{~J}$. H. Roberts, $A$ property related to completeness, Bull. Amer. Math. Soc. vol. 38 (1932) pp. 835-838. 
Moore space is a regular Hausdorff space, the sufficiency of the condition is well known. ${ }^{9}$

Theorem 4. Every metric space satisfying Axioms 0-4 of $R$. $L$. Moore's Foundations is completely (perpectly) separable.

Proof. Let $S$ be a metric space satisfying Axioms 0-4 of Foundations. No finite set of points separates $S .{ }^{10}$ Furthermore, with the help of Theorem 7 of Chapter III of Foundations it can be shown that $S$ contains no skew curve of type 1. It follows from the preceding theorem that $S$ is completely separable.

The University of TeXas

๑ P. Urysohn, Zum Metrisationsproblem, Math. Ann. vol. 94 (1925) pp. 309-315, and A. Tychonoff, Über einen Metrisationssatz von P. Urysohn, Math. Am. vol. 95 (1926) pp. 139-142. See Foundations, p. 464.

${ }^{10} \mathrm{~F}$. B. Jones, Certain consequences of the Jordan curve theorem, Amer. J. Math. vol. 63 (1941) pp. 531-544, Theorem 25. 